\title{
Zu diesem Heft: Historia magistra vitae
}

Tschou en Lai, der Spross einer jahrhundertealten Dynastie hoher chinesischer Beamter, hatte gerade sein zweijähriges Studium in Japan begonnen, als die russische Revolution ausbrach. Er vertiefte sich in die neue Politik der Bolschewiken, hörte Vorlesungen über Marx an der Universität von Kyoto und studierte intensiv das progressive linke Magazin Die neue Jugend von Tschen Du Hsiu, einem der späteren Gründer der KP Chinas. Nach seiner Rückkehr nach China begann er seine politische Karriere als Redakteur einer progressiven Studentenzeitschrift (Tianjin Student Union Bulletin) und Organisator einer Bewegung zum Boykott japanischer Waren. Er wurde verhaftet, kam jedoch bald wieder frei und ging 1920, mit einem Stipendium und elterlichem Budget ausgestattet, nach Frankreich. Von dort aus besuchte er England und Deutschland, wo er sich in Göttingen und Berlin aufhielt. In einem Brief an seinen Cousin schrieb Tschou über seine Studien: Er wolle in Europa die sozialen Fragen studieren und die Art und Weise, wie man sie politisch behandele, um die gelernten Lektionen später in China anzuwenden. Wieder zurück in China, schloss er sich 1924 der Kommunistischen Partei an, arbeitete im Widerstand gegen die japanische Besatzung und begleitete Mao Tse Tung beim Langen Marsch. Von der Gründung der Volksrepublik China an bis zu seinem Tod war er Premierminister.

Tschou en Lai war nie ein Freund radikaler Entscheidungen, er hielt Augen und Ohren offen und suchte nach einem Weg, der zum Ziel führt, ohne starke Widerstände und Ressentiments hervorzurufen. Während der Kulturrevolution war es Tschou en Lai, der einige der größten Schäden abwenden konnte und dem es gelang, eine Reihe von Persönlichkeiten vor dem Furor der Roten Garden zu retten. Dabei geriet er selbst in ihr Visier, stammte er doch aus einem Geschlecht eben jener Mandarine, die auszurotten sie sich vorgenommen hatten. Er musste Demütigungen einstecken und überlebte die Anfeindungen nur knapp. Es waren jedoch gerade Tschous politisches Geschick und seine diplomatischen Fähigkeiten, die ihn bald unentbehrlich für Mao werden ließen, denn Mao, der seine bäurische Herkunft nicht verbergen konnte, hatte während der Wirren der Kulturrevolution erkannt, dass nur Tschou en Lai in der Lage war, die chinesische Nation vor dem Auseinanderbrechen zu bewahren. Henry Kissinger, der oft und lange mit der chinesischen Führung verhandelt hat, nannte ihn eine beeindruckende Figur und beschrieb die Konstellation: »Mao dominierte jede Sitzung, Tschou erhellte sie. Mao suchte mit Leidenschaft jede Opposition zu überwältigen, Tschou war bestrebt, Opposition intellektuell zu überzeugen oder sie auszumanövrieren. Mao war sardonisch, Tschou durchdringend. Mao hielt sich selbst für einen Philosophen, Tschou betrachtete sich als Intendanten und Vermittler. Mao war eifrig bestrebt, die Geschichte zu beschleunigen, Tschou war zufrieden, wenn man ihre Verläufe ausnutzen konnte.$^{1}$

1 Kissinger, Henry 2011. On China. New York: Penguin, S. 143.

Leviathan, 42. Jg., 3/2014, S. 307-rir.317 
Aus einer dieser Unterhandlungen stammt ein denkwürdiges Bonmot. Als man Tschou en Lai fragte, was er über die Französische Revolution denke, antwortete er: »Es ist noch zu früh, darüber ein Urteil abzugeben «. ${ }^{2}$ Es scheint Geist vom Geiste Tschou en Lais, wenn man in China wieder mit Eifer Tocqueville liest, wie unsere Autorin Nele Noesselt berichtet: Reform oder Revolution? Was ist zu tun, um die wirtschaftlichen Erfolge nicht zu gefährden und doch die Ungleichheit abzumildern, die Einheit des Landes zu bewahren und zugleich die Herrschaft der KP zu festigen? Das ist die Frage, die das Politbüro beschäftigt - und dazu sucht es Antwort bei Tocqueville.

Unser Autor Eckart Reidegeld hat den Versuch unternommen, das »aus der Geschichte lernen « auf die Umweltproblematik anzuwenden. Bisher ging man zurück zu den Anfängen eines Umweltbewusstseins im 19. Jahrhundert, das sich in Heimat- und Naturschutzvereinen organisierte, oder zu den Anfängen der ersten politischen Umweltschutzbewegung in den USA in den 1930er Jahren. Nun zeigt Reidegeld, dass man auch schon vor der Industrialisierung Umweltprobleme und Ressourcenknappheit kannte und wie man damit umging - unübersehbar sind gewisse Ähnlichkeiten zu unserer heutigen Art des Umgangs mit Ressourcenverknappung. Reinhard Loske liefert in seinem Essay Ansätze, die er als Vorreiter einer nachhaltigen Entwicklung betrachtet. Dabei greift er auf den mikrohistorischen Ansatz des Lernens aus der Praxis zurück und fordert unter anderem entsprechende Bildungs- und Weiterbildungsangebote (dazu auch der Aufsatz von Harry Friebel).

Aus der Geschichte lernen ist seit alters ein Topos der Politik, Zitate antiker Autoren gehörten zum Handwerkszeug der Staatslenker und Staatswissenschaftler, und nicht zufällig ist das Latein die Metasprache nicht nur der Juristen. »Die Historie setzt uns frei, Erfolge der Vergangenheit zu wiederholen, statt gegenwärtig in frühere Fehler zu verfallen", heißt es bei Diodorus Siculus. Und die Historie, so

2 Um diese Bemerkung von Tschou en Lai ranken sich Legenden: War es Kissinger? Oder war es Nixon, der Tschou diese Frage stellte, um das Eis zwischen ihnen zu brechen, während er bei dem ersten Treffen mit dem Ministerpräsidenten durch die Anlagen der Verbotenen Stadt spazierte? Der ehemalige amerikanische Diplomat Charles W. (Chas) Freeman Jr., der damals als Dolmetscher fungierte, lieferte bei einer Konferenz anlässlich des Erscheinens von Kissingers On China eine eigene Darstellung: »Ich erinnere mich an den Gedankenaustausch zwischen Nixon und Tschou. Während Nixon jedoch nach der Bedeutung der Französischen Revolution fragte, soll Tschou bei seiner Antwort »les évènements " gemeint haben, also die Studentenrevolte der 1968er. Daraus wurde eines jener unschätzbaren Missverständnisse, an deren Auflösung niemand Interesse hat. Insofern bedauerte er auch, diese Geschichte zu enträtseln. Aber dass die Chinesen einen weiten geschichtlichen Horizont haben, während der Westen nur kurzfristiges Denken kenne, sei dennoch kein Klischée, denn es sei bekannt, dass die Chinesen sich über ihre wachsende ökonomische Stärke viel weniger wundern als der Westen: Für China gehe es nur darum, ein dreihundertjähriges Zwischenspiel des Westens zu beenden. Siehe http://mediamythalert.wordpress.com/2011/06/14/too-early-to-say-zhou-was-speaking-about-1968not-1789/ (Zugriff vom 289.07.2014). Dank an Fred Keicher für diesen Hinweis. 
meint Reinhart Koselleck, galt für rund zwei Jahrtausende als eine Schule, ohne Schaden klug zu werden. ${ }^{3}$

Doch es bestand seit jeher die Gefahr, dass eine Lehre der Geschichte missbraucht wird oder zur Blindformel verkommt und die Diskontinuität des Erfahrungsraums unterschätzt oder auch überschätzt wird. Deshalb hatte Cicero, der die Formel geprägt hat, darauf bestanden, dass nur ein Orator in der Lage sei, »der das Leben belehrenden Historie Unsterblichkeit zu verleihen, ihren Erfahrungsschatz zu perennieren ${ }^{4}{ }^{4}$

Vom Missbrauch der Geschichte berichtet Reinhart Koselleck in einer Begebenheit aus dem Jahre 1811, die Friedrich von Raumer, Historiograph der Hohenstaufen und Sekretär Hardenbergs, in seinen Erinnerungen aufgeschrieben hat: »Bei einer in Charlottenburg gehaltenen Beratung verteidigte der Sektionschef im Finanzministerium Oelssen lebhaft die Ausfertigung vielen Papiergeldes, um damit Schulden zu bezahlen. Als alle Gegengründe nicht anschlugen, sagte ich (meinen Mann kennend) mit übergroßer Kühnheit: ‘Aber Herr Geheimer Staatsrat, erinnern Sie sich doch, daß schon Thukydides erzählt, wie große Übel entstanden, weil man in Athen zuviel Papiergeld gemacht hatte. - >Diese Erfahrung<, erwiderte er bestimmend, ,ist allerdings von großer Wichtigkeit $<,-$ und so ließ er sich bekehren, um den Schein der Gelehrsamkeit festzuhalten ${ }^{5}{ }^{5}$

Hier wurde das »Lernen aus der Geschichte « zum rhetorischen Mittel, denn von Raumer wusste, dass die Antike niemals Papiergeld gekannt hatte, aber er riskierte eine Lüge, weil er ahnte, dass er Oelssen damit umstimmen konnte. Macchiavelli forderte seine Leser auf, die Alten nicht nur zu bewundern, sondern nachzuahmen. Das führt weg vom Lernen zu dem, was Blumenberg (mit Koselleck) »Präfiguration « nannte, ${ }^{6}$ nämlich der bewussten Selektion eines politischen Mythos, in dessen Nachfolge man einen Politiker aus propagandistischen Erwägungen herausstellt: »Napoleon zieht nach Ägypten aus fast keinem plausiblen Grund, mit der bloßen Platitüde, nach der Identifikation der Revolutionäre mit Brutus müsse nun er sich mit Caesar und Alexander identifizieren, diesem nach vielleicht den Zug bis nach Indien durchhalten. Noch unbegreiflicher ist, daß der, der sich bei Nacht und Nebel und unter Verletzung jeder Loyalität von seinem Heer fluchtartig aus Ägypten wegstahl, noch einmal ein an Größe vergleichbares Abenteuer suchte und nicht weniger leichtfertig

3 Koselleck, Reinhart 1989. »Historia Magistra Vitae«, in Reinhart Koselleck: Vergangene Zukunft. Zur Semantik geschichtlicher Zeiten, S. 38-66, hier S. 38. Frankfurt a. M.: Suhrkamp.

4 Ebd., S. 40.

5 Raumer, Friedrich von 1861. Lebenserinnerungen und Briefwechsel, Teil 1. Leipzig: F.A. Brockhaus, S. 118, zitiert in Koselleck 1989, a. a. O., S. 38.

6 Der Begriff Präfiguration stammt ursprünglich aus der Bibelexegese und meint »Erfüllung « einer Prophezeiung, »eine das Vorbild zur Vollendung steigernde Nachfolge« (Blumenberg 1989., a. a. O., Nachwort der Herausgeber, S. 140).. Klassisch dazu: Auerbach, Erich 1938. "Figura ", in Archivum Romanicum 22, S. 436-489; in der germanistischen Literaturwissenschaft dann von Albrecht Schöne aufgenommen: Schöne, Albrecht 1958. Saekularisation als Sprachbildende Kraft. Studien zur Dichtung deutscher Pfarrersöhne. Göttingen: Vandenhoeck \& Ruprecht. Dank an Carsten Dutt für diesen Hinweis. 
durchführte, als er nur wegen Rußlands Unbotmäßigkeit gegen die Kontinentalsperre auf Moskau zumarschierte. Dort ließ er sich im Kreml nieder und vergaß über der Selbsterhebung, die ihn das erleben ließ, die Einwohner der Stadt an der massenweisen Brandstiftung zu hindern «. ${ }^{7}$

Dilettantismus nennt Blumenberg dieses Vorgehen, denn als gelernter Soldat war Napoleon zwar in der Lage, mit einem »ungeheuren, atlashaften Gedächtnis « seine Freunde und seine Feinde zu verblüffen, aber seine Unternehmungen hatten »unerlernbare Voraussetzungen «: Während die räumliche Kontinuität dem Soldaten die Anwendung der erfolgreichen Rezepte aus der Geschichte zu erlauben scheint, ist ein solches Verfahren im sozialen Diskontiuum nicht ohne Berücksichtigung der Veränderung von sozialen Kräften sinnvoll. Die Tradierbarkeit des Topos vom Lernen aus der Geschichte war an eine »tatsächliche Konstanz jener Vorgegebenheiten « (Koselleck) gebunden, die eine potenzielle Ähnlichkeit irdischer Ereignisse zuließen. Und wenn sozialer Wandel stattfand, dann so langsam und so langfristig, dass die Nützlichkeit vergangener Beispiele erhalten blieb. Der Wechsel zum 19. Jahrhundert erst brachte die Beschleunigung des Wandels mit sich, die das Lernen aus der Geschichte erschwerte und komplizierte.

Hatte Napoleon geglaubt, nur die französische Gesellschaft habe sich revolutioniert, ein Wandel, den er nun auf die ganze Welt übertragen müsse? Wie konnte, räsoniert Blumenberg, eine der zivilsten europäischen Nationen diese billigen Ideen für groß halten? Und wie konnte es geschehen, dass diese Denkart in ihrer ganzen Dürftigkeit wiederzufinden ist in den Monologen im Führerhauptquartier?

Goebbels sah in Hitler die Wiederkehr der Gestalt Friedrichs des Großen, ein Präfigurativ, das mehr einer Reklamephantasie glich als einer nüchternen Annäherung an historisches Lernen. »Die Bezugnahme auf Präfiguranten stärkt nämlich nur die, die sich als Präfigurate empfinden ", so Blumenberg, sie muss jedoch dann versagen, wenn der Abstand zwischen der historischen Figur und der aktuellen Politik desjenigen, der sich in ihr wiederspiegeln zu können meint, unüberbrückbar wird. Göbbels hatte am 30. Januar 1945 zum Tage der Machtergreifung gerade den in zweijähriger Arbeit mit großem Aufwand hergestellten Film »Kolberg « in die Kinos gebracht, in dem es um die heroische und erfolgreiche Verteidigung Kolbergs im Jahre 1807 gegen die Franzosen ging, doch da standen die russischen Armeen bereits $60 \mathrm{~km}$ östlich von Berlin. Und Göbbels sinnierte weiter: »Unsere Redner in den Versammlungen setzen sich nicht mehr richtig durch. Die Argumente, die nur auf historische Beispiele verweisen, ziehen nicht mehr", schrieb er am 17. März 1945 in sein Tagebuch und verbot die Veröffentlichung der Meldung über den Fall Kolbergs. Blumenberg notiert, wie sich langsam auch bei Goebbels der Mythos um den Führer auflöst: »Am 28. März eine schlimme Eintragung: das Bild Hitlers hat sich aus den Umrissen des Preußenkönigs gelöst, er ist nicht der Mann, aus richtigen Erkenntnissen die richtigen Schlüsse zu ziehen: Darin unterscheidet er sich meines Erachtens in starker Weise von Friedrich II «. ${ }^{8}$

7 Blumenberg, Hans 2014. Präfigurationen. Arbeit am politischen Mythos. Berlin: Suhrkamp, S. 56.

8 Blumenberg 2014, a. a. O., S. 46. 
Wenn also unser Autor Siegfried Schieder die Anerkennung einer deutschen Führungsrolle in der EU anspricht, so verkennt er nicht die Schwierigkeiten, die die Präfigurationen dieser Rolle Deutschlands für Europa mit sich bringen: Ist die Gefahr des ökonomischen Dilettantismus und der Geschichtsvergessenheit in diesem Unternehmen nicht möglicherweise größer als die Gefahr, dass Europa das Ziel der Vereinheitlichung ohne eine politische Führerschaft verpasst?

Friedrich der Große nennt die Geschichte die Schule der Herrscher - er selbst habe seine Kombinationskraft durch ständiges Vergleichen früherer Fälle geschärft. Aber vielleicht war Friedrich der letzte europäische Fürst, der die Freiheit, zu agieren, aus der Gewissheit nahm, dass sich die "Szenen der Weltgeschichte wiederholen, man brauche nur die Namen auszutauschen ", dass also das Machtspiel nur mit den bekannten Gegenspielern zu rechnen hatte. Die Französische Revolution hat neue Spieler hervorgebracht, und der »überschaubare Raum der europäischen Fürstenrepublik « endete 1789. Seither zeigt sich im Lichte dieser Erfahrung eine geballte Skepsis gegen die Vorstellung, dass Regierungen oder Völker aus der Geschichte lernen können: ${ }^{9}$ Die Unüberschaubarkeit der Geschichte nahm zu, die Möglichkeit, aus ihr zu lernen, schien verbaut angesichts ihrer Ungewissheiten und Kontingenzen, und zugleich dekretierten die französischen Revolutionäre eine "neue Zeit ", die mit der Vergangenheit vollständig gebrochen habe. Dass die Befreiung von der Vergangenheit nicht gelang und nicht gelingen konnte, das hat schon Tocqueville gezeigt.

Das Lernen aus der Geschichte scheint in unseren Breiten mehr und mehr aus der Mode gekommen, vorgedrängt hat sich der historisch flache Vergleich: »Best practice " ist das Steckenpferd der europäischen Politiker und Politikberater, die Übernahme von Praktiken, die sich im synchronen Vergleich als die besseren erwiesen haben sollen. Es ist ein Methodenwandel, der erklärungsbedürftig ist: Wie kam es dazu? Die Frage ist noch nicht beantwortet, aber es häufen sich die Hinweise, dass Übernahmen aus anderen geografischen Räumen dieselbe Problematik aufweisen: Aus der Geschichte lernen ist ebenso schwierig wie die Übernahme einer fremden Praxis, denn auch solche »Unternehmungen « mit »ihren Dimensionen nach unerlernbaren Voraussetzungen « sind nicht geeignet für Dilettanten. Geografische, strukturelle und kulturelle Differenzen, historische Diskontinuitäten ebenso wie Pfadabhängigkeiten setzen jeder plagiatorischen Identifikation mit einem Präfigurativ-Modell Grenzen.

Die Formel von der » best practice « beruht jedoch nicht allein auf dem Vergleich, sondern auch auf einer ganz bestimmten Vorstellung von Zukunft, die sich aus der Gegenwart ableitet: Damit werden Erkenntnisse der Geschichtswissenschaft über Konjunkturen und die Rhythmen der langen Dauer zugunsten praktisch-politischer Anwendung ausgeblendet, und es wird quasi magisch auf die Dauerhaftigkeit der aktuellen Strukturen spekuliert. Es ist zugleich eine Unterschätzung der Diskontinuität im Verhältnis zur Zukunft und eine Überschätzung der Diskontinuität im Verhältnis zur Vergangenheit. Einer der Gründe, warum man auf das Lernen aus der Geschichte glaubt verzichten zu können, liegt in der Vergötterung des Neuen. Der Topos der Neuheit, des Nie-da-Gewesenen ergießt sich täglich aus der Reklame

9 Koselleck 1989, a. a. O., S. 58 f.

Leviathan, 42. Jg., 3/2014 
in Augen und Ohren, und so ganz blieb kein Feld davon verschont: Hatte man nicht behauptet, dass es im neuen digitalen Zeitalter keine Krisen mehr geben werde, da alle Informationen allen und jederzeit zur Verfügung stünden? Man nannte es eine Revolution und griff damit auf das gesamte Bedeutungsfeld der Französischen ebenso wie der Russischen Revolution zurück: Die Schaffung des neuen Menschen und die Ablösung von den Krisen der alten Ökonomie wurde gepredigt. Wie also sollte sich eine Blase aufbauen, wenn jeder sich über den Zustand der Börsen und des Immobilienmarktes informieren kann? Und doch fiel der dot.com-Markt 2001 in sich zusammen, nachdem er von einer der wirksamsten Propagandamaschinerien der Neuzeit, nämlich derjenigen der Finanzbranche, aufgeblasen worden war. Und trotz dieser Widerlegung schien auch keiner Notiz zu nehmen von der nächsten Gefahr, erkennbar schon am bedenklichen Anstieg der Zahl der Häusermakler. ${ }^{10}$ Alles deutete auf die Bildung einer Blase hin, warnende Stimmen aber gingen unter. Der Hypothekenmarkt wurde zum Saugstutzen für einen sich aufbauschenden Markt für Verbriefungen: Die Darlehen wurden von den Hypothekenbanken nicht gehalten, sondern auf einem neuen Kreditmarkt verkauft und gerieten in das Visier der Finanzbranche, die stets auf der Suche nach Vermögensanlagen ist. So strebten Bear Stearns, Lehman Brothers, Merrill Lynch, Goldman Sachs, J.P. Morgan, Deutsche Bank, die schweizerische UBS und die Credit Suisse mit sogenannten Zweckgesellschaften in diesen neuen Markt, die den Hypothekenbanken die Darlehensverträge abkauften, sie "verbrieften " und in den Handel brachten als angeblich sichere Papiere - sogenannte $\mathrm{ABS}^{11}$-, die hohe Erträge insbesondere in der zweiten Phase bringen sollten, wenn die Zinsen für die Hypotheken wieder stiegen. Diese »innovativen Finanzprodukte « wurden in Tranchen gestückelt und an Investoren und Sparer verkauft (emittiert). Aber niemand wusste, was in den Papieren versteckt war - es gab keine Transparenz, da half auch das Internet nicht, denn dort lässt sich nur das ablesen, was zuvor eingestellt wird.

Wir wissen, wie die Geschichte weiterging: Solange der Hypothekenmarkt von steigenden Immobilienpreisen gekennzeichnet war und die Zinsen niedrig blieben, lief die Maschinerie rund. Der Markt für ABS und die darauf beruhenden kurzfristigen Kredite (CPs) wuchs und erreichte in den Jahren 2005 und 2006 jeweils ein Volumen von 900 Milliarden US-Dollar. Insbesondere die Überbrückungskredite, die die Banken den Hedgefonds für ihre Übernahmen gewährten, beruhten auf ABS. Doch seit Beginn der Krise sanken die ABS-Werte, und bald wurde der Handel ganz ausgesetzt. Ohne eine sichere Wertpapierbasis aber ließen sich jetzt keine kurzfristigen Kredite mehr vergeben. Die Propagandamaschine hatte kritische Stimmen marginalisiert, und die Kontrollinstanzen versagten völlig, ja, die US Börsenaufsicht SEC übernahm sogar die Software der Investmentbanken, um die Risiken der aufgeblasenen Volumina zu überprüfen. Der Absturz war nicht mehr zu verhindern -

10 Im Jahre 2005 stieg sie bereits auf 400.000 an, wie die New York Times am 8. Oktober berichtete: Schon 50.000 Firmen seien mit der Vermittlung von Hypothekenverträgen beschäftigt, ein ganz neuer Berufszweig habe sich etabliert, den es vor 25 Jahren noch gar nicht gegeben habe.

11 ABS: »asset-backed securities «, vermögensgesicherte Wertpapiere. 
was aber hinderte die politische Klasse, aus der Geschichte der Krisen zu lernen? Die Chinesen aber lernten aus der Finanzkrise, »dass die kapitalistischen Lehrmeister ebensolche Dilettanten waren wie ihre chinesischen Eleven «. ${ }^{12}$

Ob es tatsächlich mehr Transparenz gibt im digitalen Zeitalter? Das ist gar nicht die Frage, sondern wie die Nachrichten und Informationen bewertet und gestreut werden. Und da gelten die klassischen Regeln der Rhetorik. So wird es auch immer Bereiche geben, in denen Transparenz die Lage nicht verbessert, sondern verschlechtert: Die Behauptung, ein Geschäftskunde sei am Rande der Insolvenz, führt notwendig zur Insolvenz, denn niemand wird mit einem solchen Kaufmann mehr Geschäfte machen. Ohne eine solche Rufschädigung aber hätte die Chance bestehen können, einer drohenden Insolvenz wieder zu entkommen.

Wie verhält es sich mit der Transparenz in der Politik? Könnte ein Übermaß an Transparenz nicht der Demokratie schaden? Das fragt unser Autor Max-Otto Baumann. Leiten bislang die Journalisten und Redakteure der Zeitungen durch den täglichen Dschungel der Nachrichten, so bietet das Internet - zumindest auf den ersten Blick - einen direkten Zugang zu den neuen Botschaften des Tages. Das dort sichtbar Werdende erweist sich jedoch für die große Mehrzahl als überkomplex und als für den Alltagsgebrauch kaum noch bezwingbare Fülle. Und da die Bewertung der Bedeutung von Ereignissen damit nicht leichter fällt, zeigt sich zugleich das, was unser Autor ein »epistemologisches Paradoxon " nennt: »Je schärfer ein Detail betrachtet wird, desto mehr geraten die Strukturen aus dem Blick. Im Rasterelektronenmikroskop erkennt man Atome, aber nicht mehr, welches lebensweltliche Objekt sie bilden. So müssen die politischen >Rohdaten`, welche durch Transparenz ans Licht kommen, erst wieder zu politischem Sinn kombiniert werden - ein anspruchsvoller Prozess der Kontextualisierung, bei dem einiges schiefgehen kann." Die Folge ist, dass statt der Journalisten als Pfadfinder nunmehr die Angebote der Internetunternehmen diese Rolle spielen: Nachrichten bieten die Anbieter in eigener Auswahl an. Es sind Kanäle, deren Transparenz eher geringer ist als diejenige der klassischen Zeitungen.

Die von der Piratenpartei gewünschte »liquid democracy « beruht auf dem Vertrauen in die Differenzierungsfähigkeit ihrer Bürger. Aber daran zweifelten die Mütter und Väter des Grundgesetzes und bauten, so gibt Baumann zu bedenken, zahlreiche Prinzipien des institutionalisierten Misstrauens in die Verfassung ein Gewaltenteilung, Opposition, Misstrauensvotum etc. Nicht Vertrauen, sondern Misstrauen und kritische Distanz seien die angemessenere Haltung in der Demokratie mit ihren offenen und versteckten Interessenkämpfen.

Reform oder Revolution? Wenn man liest, was Tocqueville über die Physiokraten schreibt, bekommt man eine Ahnung, was Tschou en Lai mit seiner Antwort gemeint haben könnte: Nicht nur, dass die Physiokraten gerne auf ein Land verwiesen, das zu ihrer Zeit höchstes Ansehen unter den Aufklärern genoss: »Ich übertreibe nicht, wenn ich versichere, daß es ihrer nicht einen gibt, der nicht an irgend einer Stelle seiner Schriften eine emphatische Lobrede auf China gehalten

12 Erber, Georg 2014. »China: Land der unbegrenzten Möglichkeiten? ", in Leviathan 42, 1, S. 30-69, hier S. 48.

Leviathan, 42. Jg., 3/2014 
hätte «. ${ }^{13}$ Überraschend auch, dass »die umstürzlerischen Theorien, die man heutzutage Sozialismus genannt hat ", nicht neuen Ursprungs sind, sondern bereits bei den ersten Physiokraten zu finden sind. Tocqueville empfiehlt die Lektüre von Morellis " Gesetzbuch der Natur ", in welchem sich jene politischen Theorien finden, »welche Frankreich in den letztvergangenen Jahren am meisten erschreckt haben «: "Nichts in der Gesellschaft soll jemand besonders oder als Eigentum gehören «, sagt der erste Artikel jenes Gesetzbuchs. »Das Eigentum ist abscheulich und derjenige, der es wieder einzuführen versuchen wird, soll wie ein rasender Narr und als Feind der Menschheit zeitlebens eingesperrt werden. « "Jeder Bürger wird auf öffentliche Kosten ernährt, unterhalten und beschäftigt werden «, sagt der zweite Artikel. »Alle Erzeugnisse werden in öffentlichen Magazinen ausgespeichert, um unter alle Bürger verteilt zu werden und ihren Lebensbedürfnissen zu dienen. Die Städte sind nach dem gleichen Plane zu bauen; alle Wohnungen der Privatpersonen sollen gleichartig sein. Mit fünf Jahren werden alle Kinder der Familie genommen und gemeinschaftlich auf Kosten des Staates in gleichförmiger Weise erzogen. «14

Wie zeitgenössisch sind diese Theorien? Klingen solche Sätze in den Ohren chinesischer Politikbüromitglieder nach tua res agitur? Wie nahe ist das Frankreich der Physiokraten - und das Tocquevilles - den politischen Fragen des heutigen China? Tocqueville meint, bei den Physiokraten ein starkes Interesse für die Gleichheit, aber ein eher schwaches Interesse für die »Freiheit " ausmachen zu können: In den Büchern der Physiokraten erkenne man schon »jenes revolutionäre und demokratische Temperament, ${ }^{15}$ das uns gut bekannt ist; sie hassen nicht nur gewisse Privilegien, sondern alle Verschiedenheit überhaupt ist ihnen verhasst; sie würden die Gleichheit selbst noch in der Knechtschaft anbeten; was sie in ihren Plänen stört, verdient nur vernichtet zu werden. Verträge flößen ihnen wenig Respekt ein, Privatrechte gar keine Achtung; vielmehr gibt es in ihren Augen eigentlich schon keine Privatrechte mehr, sondern nur Gemeinnutz [...]

Sie haben die Idee zu allen sozialen und administrativen Reformen, welche die Revolution bewerkstelligte, bereits gehabt, bevor der Gedanke an freie Institutionen in ihrem Geiste noch zu dämmern begann. Sie sind allerdings dem Getreidefreihandel, dem laisser faire und laisser passer in Handel und Industrie sehr günstig; an die eigentlichen politischen Freiheiten denken sie gar nicht [...] Die meisten von ihnen zeigen sich zuerst als entschiedene Gegner der beratenden Versammlungen, der örtlichen und untergeordneten Gewalten und überhaupt aller jener Gegengewichte, die zu verschiedenen Zeiten bei allen freien Völkern eingeführt worden sind um der Zentralgewalt die Waage zu halten. `Das System der Gegenkräfte‘, sagt Quesnay, >ist in einer Staatsverfassung eine verderbliche Idee. > Die Spekulationen, nach welchem man das System der Gegenkräfte ersonnen hat, sind utopisch‘, sagt ein Freund

13 Tocqueville, Alexis de 1935, »Das Ancien Régime und die Revolution «, in Autorität und Freibeit. Schriften, Reden und Briefe, ausgewählt und eingeleitet von Albert Salomon, S. 293. Zürich: Rascher.

14 Tocqueville 1935, a. a. O., S. 294-295.

15 Ob die Sozialisten Demokraten sind oder nicht, das überlässt Tocqueville den Etymologen; vgl. ebd. 
Quesnays. Die einzige Garantie, die sie gegen Mißbrauch der Gewalt erfinden, ist die staatliche Erziehung, denn, so bemerkt ebenfalls Quesnay, >der Despotismus ist unmöglich, wenn die Nation aufgeklärt ist . «16 $^{16}$

Quesnay, das Haupt der Physiokraten, war ursprünglich Leibarzt der Madame Pompadour und hatte zugleich den Rang eines vierten Leibarztes des Königs. Seine Versuche, den König für volkswirtschaftliche Themen zu erwärmen und ihm die Notwendigkeit der Verbesserung der Lage der Bauern nahezubringen, sind bekannt ${ }^{17}$. Wenn er sich für den Freihandel aussprach, so deshalb, weil er glaubte, die Getreidepreise würden steigen, wenn sie nicht mehr amtlich festgelegt würden: Ein solches Ergebnis würde die Lage der Bauern verbessern. Von ihm konnte man also keine Begeisterung für den Königsmord erwarten, und so waren auch seine Jünger - von Mirabeau dem Älteren über DuPont de Nemours bis zu Turgot - keine Palaststürmer, sondern sie erwarteten alle Verbesserungen vom Monarchen.

Und weist Tocqueville nicht auch deutlich auf ein Problem hin, das China plagt, nämlich den schwierigen Umgang mit den immer zahlreicher werdenden Millionären, die kein Verständnis mehr für Politik haben? »In der Tat gibt es einen sehr gefährlichen Abschnitt im Leben der demokratischen Völker. Wenn die Freude an materiellen Genüssen sich bei einem dieser Völker schneller entwickelt als die Bildung und die Gewohnheiten der Freiheit, dann kommt ein Augenblick, in dem die Menschen beim Anblick dieser neuen Güter, nach denen sie schon die Hände ausstrecken, hingerissen und wie von Sinnen sind. Nur von dem Bestreben erfüllt, sich Reichtum zu erwerben, sind sie sich nicht mehr des engen Bandes bewußt, das den persönlichen Reichtum eines jeden von ihnen mit dem Wohlstand aller verknüpft. [...] Diese Leute glauben sich an die Lehre vom Interesse zu halten, machen sich aber davon nur eine verschwommene Vorstellung und vernachlässigen, um ihren Geschäften besser nachgehen zu können, das wichtigste von allem, nämlich: Herr über sich selbst zu bleiben. «18

Geschichte hält viele Lehren vor, sie aufzubereiten ist heute nicht mehr die Aufgabe des Orators, sondern seit der Aufklärung das Geschäft der Philosophen und der Geistes- und Gesellschaftswissenschaften. Das Ancien Régime behandelte die Geistes- und Staatwissenschaften als geistvolle und achtenswerte Betätigung des Menschengeistes, die Revolutionsdiktatur erstickte sie und unterdrückte ihre Autoren oder brachte sie auf die Guillotine. Napoleon betrachtete die » Klasse für Staatsund Geisteswissenschaften ", wie die Akademie sich seinerzeit nannte, mit misstrauischen Augen, und die Klasse, die aus berühmten Persönlichkeiten zusammengesetzt war, reagierte, indem sie sich in Vergessenheit zu bringen suchte: Man befasste sich mit der Zeit der Pharaonen, mit dem Instinkt, und in der Volkswirtschaft beschäftigte man sich mit dem Steigen und Sinken des Pegels der Seine.

16 Ebd., S. 289.

17 Vgl. dazu Lüthy, Herbert 1959. Francois Quesnay und die Idee der Volkswirtschaft. Reihe »Kultur- und staatswissenschaftliche Schriften « der Eidgenössischen Technischen Hochschule. Zürich: Polygraphischer Verlag, S. 6.

18 Tocqueville, Alexis de 1954. Das Zeitalter der Gleichheit, hrsg. v. Siegfried Landshut. Stuttgart: Kröner Verlag, S. 61. 
Doch vergeblich: Napoleon bemerkte sie trotzdem, und als er »die allerletzten Spuren der staatsbürgerlichen Freiheiten vernichtet hatte", vernichtete er auch die Freidenker in einem Dekret »durch stillschweigende Übergehung «. ${ }^{19}$

Heute stehen die Geistes- und Gesellschaftswissenschaften wieder in Blüte. Aber sie geraten unter Druck. Dabei sind die Politiker nicht mehr wie Napoleon getrieben von der Angst vor den Gefahren der Geistes- und Sozialwissenschaften, sondern von einem anderen Motiv: Sie verlangen Innovationen ${ }^{20}$ und einen Nachweis ihrer Nützlichkeit. Es gilt also zu zeigen, was die Geistes- und Sozialwissenschaften beitragen können. Deshalb hat der Satz von der historia magistra vitae eine neue Form bekommen: "Was lernen wir daraus? « muss am Ende jedes Aufsatzes beantwortet werden, das Fazit legt nahe, wie nützlich es sei, was man aus der jeweils erzählten Geschichte gelernt hat. Für die Regierenden muss der Geistes- und Sozialwissenschaftler wieder zum Orator werden.

In der Debatte um die künftige Orientierung der Stadtsoziologie verteidigt Johanna Hörning den Anspruch der Dritten Welt auf Gehör: Stadtsoziologie dürfe sich nicht auf das Modell der europäischen Stadt allein beschränken. In ihrer Kritik greift sie auf das begriffliche Paradoxon der Debatte zu: Was ist die Essenz der Stadt? Dass es keine »Essenz " gibt, sondern dass Stadt durch Heterogenität und Simultaneität gekennzeichnet sei - Stadt sei der Raum der Widersprüchlichkeit.

Jörg Dürrschmidt weist auf die Kluft zwischen einem globalen Ansatz, der einer großen Theoriebildung folgt, und dem eher theoriefeindlichen Mikroansatz beschreibender Erkundungen des urbanen Alltags hin, die gelegentlich zu einer Zerreißprobe werde, sowohl für die städtische Wirklichkeit (die er an den zwei Beispielen London und Wittenberge untersucht hat) als auch für die Theorie. Der Eigenlogik-Ansatz, das ist seine Kritik, verorte sich zum einen jenseits der Logik übergeordneter Superstrukturen, verweigere sich jedoch zum anderen auch »jener Konkretionslogik, der angesichts des Details kleinräumiger Vergemeinschaftungen ein Blick für das städtische Ganze verloren gehe«.

Das Rolltreppengleichnis, nach dem die Bevölkerung Londons sich stets auf dem Weg nach oben fühle, während sich die Bevölkerung Wittenberges auf der anderen Treppe - auf dem Wege nach unten - wähne, wird man als Gegenwartsdiagnose der Befindlichkeiten der Bewohner verstehen. Eine längerfristige Studie würde diese Diagnose um Generationserfahrungen bereichern können, Änderungen der Verteilungsmuster über lange Dauer (longue durée) zeigen und sichtbar werden lassen, dass der »Weg nach unten " oder der »Weg nach oben « Momentaufnahmen im historischen Raum darstellen. Raum und Zeit verändern sich in der longue durée,

19 Ebd., S. 148.

20 Peter Sloterdijk sieht darin eine große Gefahr: »Das Lernen bedeutete früher etwas ganz anderes als heute: Aneignung von Überlieferung, das ewige Spiel von Innovation und Wiederholung. Inzwischen zählt nur noch Innovation. Die ganze Welt ist eine große Testanlage für die Verträglichkeit von Innovationen. Mit der Kernkraft haben wir einen solchen Test dramatisch durchexerziert «; Sloterdijk, Peter 2014. »Interview: Diesmal machen wir die Katastrophe selber", in Handelsblatt vom 18./19./20. Juli 2014. Dort gibt er auch fünf Bücher an, die ein Wirtschaftsführer und Entscheider gelesen haben sollte, darunter Alexis de Tocquevilles Buch Der alte Staat und die Revolution. 
und so, wie London mit steigendem Meeresspiegel unter Wasser geraten wird und sich eine andere Lebensweise und ein anderes Grundmotiv einstellen werden, mag sich auch für Wittenberge mit anderen Umständen eine andere Lebensmotivation zeigen. Aus der Geschichte lernen heißt auch, sich nicht von der Gegenwart gefangen nehmen zu lassen.

Reinhard Blomert 